\title{
Self Consistently Generated Charge Cylinder in BETA Device
}

\author{
Rajwinder Kaur, A. Sarada Sree, Shiban Kishan Mattoo \\ Institute for Plasma Research, Gandhinagar, India \\ Email: nikky@ipr.res.in
}

Received August 22, 2012; revised September 20, 2012; accepted September 27, 2012

\begin{abstract}
This paper presents a study on near cathode space charge region in BETA (Basic Experiments in Toroidal Assembly), a toroidal plasma device with purely toroidal magnetic field. A charge cylinder has been found to be embedded in the plasma center corresponding to the hot filament cathode location in poloidal cross section. This charge cylinder has been created by the primary electrons emitted from the filament surface, which in turn, leads to the formation of a potential well in the core plasma. We have proposed a model, which shows that a tiny fraction of injected energetic electrons is sufficient to sustain the observed potential well. We have examined the equilibrium of the charge cylinder in poloidal cross-section and found that it exhibits equilibrium configuration by forming circulation pattern of primary electrons. The circulation pattern is formed by vertical drift due to toroidal magnetic field and self-consistent poloidal $\boldsymbol{E} \times$ $\boldsymbol{B}$ drift. We have concluded that the self-consistency is in adjusting the poloidal drift to the vertical drift of the trapped primary electrons.
\end{abstract}

Keywords: Hot Cathode Discharge; Near Cathode Space Charge; Toroidal Magnetic Field

\section{Introduction}

The near cathode space-charge behavior is of great interest in plasma discharges [1-5]. These studies have attracted lot of interest due to their applications in plasma surface processing. Analytic and numerical models have been developed [2-5] to gain the understanding of the physical processes occurring in the cathode region of discharges. The knowledge of primary electron trajectories in this region helps in understanding and predicting various discharge characteristics. Three dimensional (3D) computer simulation techniques have been developed for tracking the primary electrons and applied to numerous applications [6-9]. The influence of different magnetic fields on plasma discharge has been studied by tracing the primary electron trajectories near the filament.

This paper presents an experimental study on near cathode space charge region in a hot filament cathode discharge produced toroidal plasma device BETA. The plasma in BETA device [9-11] is produced by a hot filament cathode discharge. The hot filament cathode injects energetic primary electrons into the plasma. However, the observed value of the charge density is much less than the injected charge density. An anomalous radial crossfield current transports most of the injected charge [1215]. Only a small amount of residual space charge still exists that is responsible for the creating a charge cylin- der in plasma center with axis coinciding with the major axis of the torus. This charge cylinder in turn, sustains the observed potential well. We have examined the equilibrium of this charge cylinder and proposed a model to account for the behavior of trapped primary electrons.

The paper is organized as follows. Section 2 gives an outline of experimental setup and experimental results are given in Section 3. Section 4 gives a discussion on the existence and equilibrium of the charge cylinder. Finally conclusions drawn from the experimental results and discussion are given in Section 5.

\section{Experimental Setup}

The experimental apparatus BETA device (Figure 1) consists of a toroidal vacuum chamber of major radius 45 $\mathrm{cm}$ and minor radius $15 \mathrm{~cm}$ pumped down to base pressure of $10^{-6}$ Torr [9-11]. A toroidal magnetic field is produced by a set of 16 coils and can be varied up to $1 \mathrm{kG}$. The plasma is produced by a discharge between a cathode of hot filament and the vacuum vessel at a pressure of $10^{-4}$ Torr. The working gas is argon. A circular aperture of diameter $18 \mathrm{~cm}$ defines the size of the plasma. The plasma lacks equilibrium in the MHD sense. However, a steady state plasma can be maintained by the balance of charge production and their loss. Time averaged plasma parameters are measured using a radially mov- 


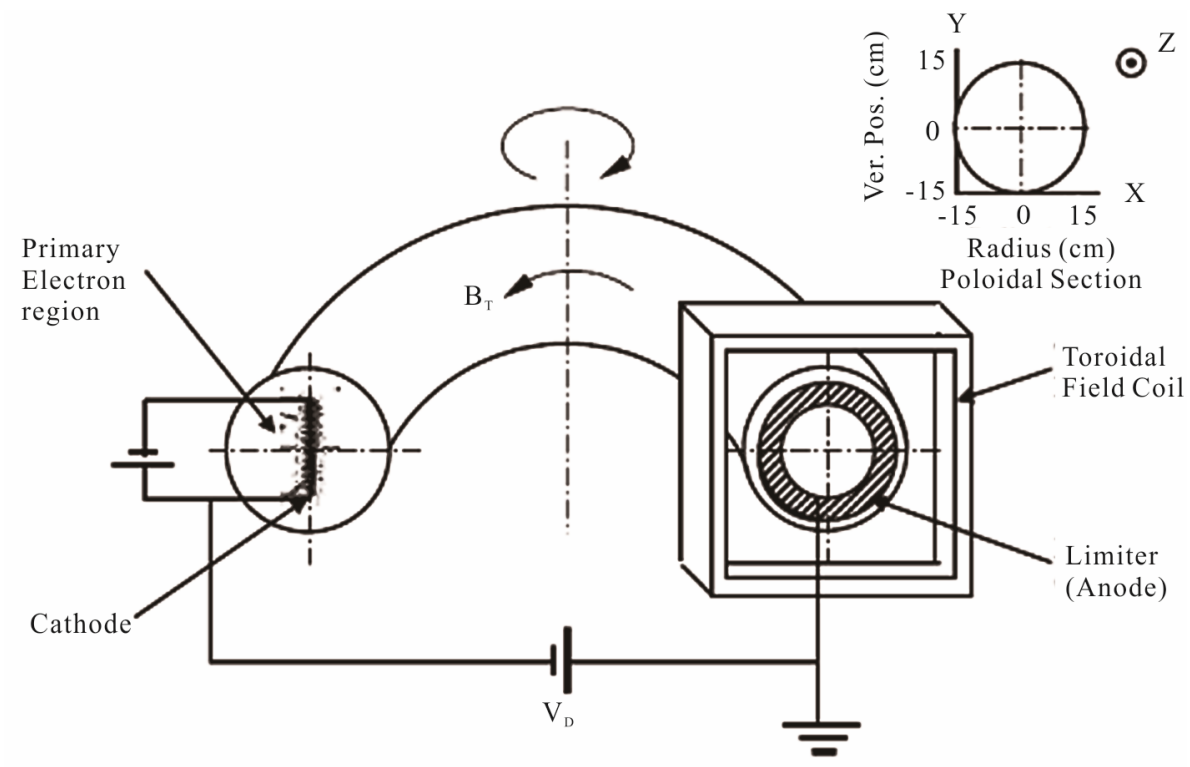

Figure 1. Schematic of BETA device.

able vertical array of 10 Langmuir probes $(l=5 \mathrm{~mm}, \phi=$ $1 \mathrm{~mm})$. This array measures the plasma parameters at different vertical locations in one-half (one side of the mid plane) of the plasma cross section at a fixed radial location. The array is, then, rotated for measurements in the other half of the plasma cross section at the same radial location. This enables the measurements from $Y=-9$ to $+9 \mathrm{~cm}$ at a fixed radial location. The array is moved radially to create a grid in XY-plane.

\section{Experimental Results}

The poloidal maps of various plasma parameters measured in the BETA device are shown in Figure 2. The typical plasma density is $\sim 10^{16} \mathrm{~m}^{-3}$. The floating potential maps show the presence of potential well at hot filament cathode location along the connecting toroidal magnetic field lines. The equipotential contours are vertically elongated near the filament. The vertical extent of the contours (with $1 / \mathrm{e}^{\text {th }}$ value of the peak) is approximately of the length of filament i.e., $\sim 14 \mathrm{~cm}$ and radial extent $\sim 3$ $\mathrm{cm}$. The contours show a net vertically downward shift $\sim 2 \mathrm{~cm}$ with respect to the filament. This direction corresponds to the drift direction of primary electrons due to gradient and curvature of toroidal magnetic field. However, the contours generally close within the limiter and do not extend indefinitely in primary electron drift direction. Beyond the $1 / 10^{\text {th }}$ value of the peak, the contours widen in major radius direction and the potential varies smoothly. The radially inward electric field estimated from plasma potential contours is $\sim 500 \mathrm{~V} / \mathrm{m}$ near the filament. The vertical electric field $\sim 1000 \mathrm{~V} / \mathrm{m}$ exists only at the filament ends and is directed towards the filament ends. As we move away from the potential well, the shapes of the plasma potential contours change from vertical ellipses to nearly circular ones. The electric field away from the potential well is directed radially inwards with magnitude of $\sim 40 \mathrm{~V} / \mathrm{m}$. The typical electron temperature in BETA device is $\sim 4-10 \mathrm{eV}$.

\section{Discussion}

The energetic primary electrons emitted from the hot filament cathode circulate along toroidal magnetic field in both the directions, thereby, constitute zero net current. As mentioned in the preceding section that the floating potential contours indicates that charge injected by the hot filament spreads as a $3 \mathrm{~cm}$ thick cylindrical charge layer of $14 \mathrm{~cm}$ length, located at the major radius of the midpoint of the filament, shown schematically in Figure 3. It forms a potential well located at the major radius of the midpoint of the filament. We call it charge cylinder and investigate its properties in the succeeding sections.

\subsection{Observed Charge Density of Charge Cylinder}

The charge density integrated over the charge layer will appear as a surface charge density $\sigma \sim-\varepsilon_{0} E_{\perp}$. For the measured electric field value of $E_{\perp} \sim-500 \mathrm{~V} / \mathrm{m}$, the estimated surface charge density is $\sigma \sim 4.43 \times 10^{-9}$ Coulombs $/ \mathrm{m}^{2}$. The primary electron density, thus calculated, varies from $\sim 1.4 \times 10^{13}$ electrons $/ \mathrm{m}^{3}$ for $2 \mathrm{~mm}$ thick (i.e. filament diameter) charge cylinder to $\sim 10^{12}$ electrons $/ \mathrm{m}^{3}$ for $3 \mathrm{~cm}$ width of the observed nearly rectangular potential contours. Comparing this value of charge density with the statistical charge fluctuation in plasma which is $\sim 2 \times 10^{8} \mathrm{~m}^{-3}$ implies that the charge density in the charge layer is at least four orders of magnitude greater than 

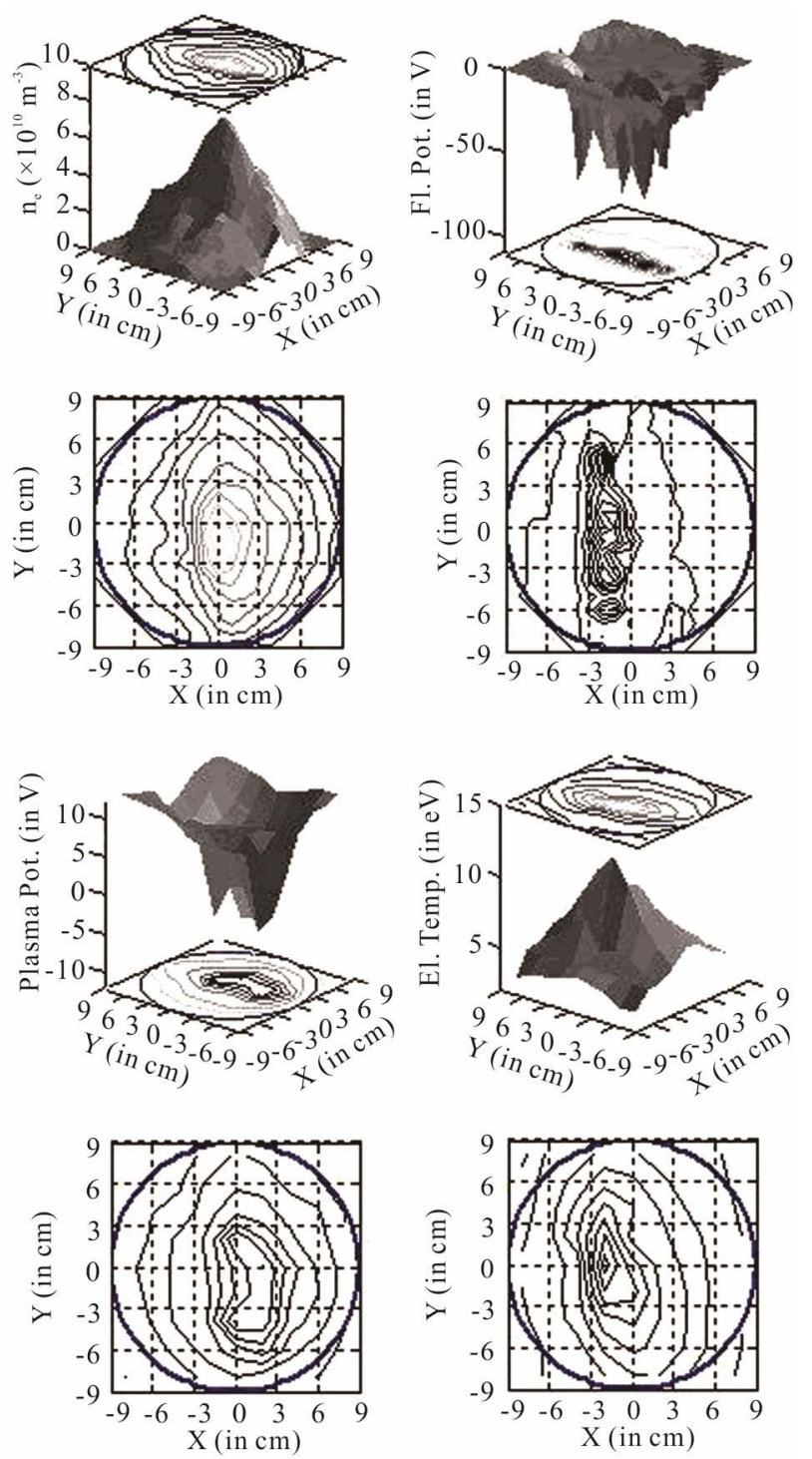

Figure 2. Poloidal maps of (a) electron density; (b) floating potential; (c) plasma potential and (d) electron temperature.

local charge fluctuations. This establishes that charge cylinder is significant and should play a crucial role in plasma dynamics of BETA device.

\subsection{Injected Charge Density}

We have estimated the injected charge by two methods, viz., by calculating the injected current due to primary electrons and from the arc current between the filament cathode and the anode of vessel wall.

\subsubsection{Injected Primary Current}

The electrons injected by the hot filament cathode form a shadow-like cylindrical layer along the connecting toroidal magnetic field lines. This cylindrical layer has the radius of $0.45 \mathrm{~m}$, height of $0.14 \mathrm{~m}$ and thickness of $2 \mathrm{~mm}$ corresponding to the location and size of the filament. The thickness of the cylindrical layer shadow expands to $\sim 3 \mathrm{~cm}$ (corresponding to several electron gyroradii) as shown in Figure 4, thereby increasing the volume of the layer.

The current carried by the primary electrons is $I_{p e}=$ $n_{p e} e v A$, where $n_{p e}$ is the density of primary ionizing electrons, $e$ is the electron charge of $1.6 \times 10^{-19} \mathrm{C}, v=4.7 \times$ $10^{6} \mathrm{~m} / \mathrm{s}$ is the velocity of $120 \mathrm{eV}$ electrons and $A$ is the area of electron emission. The current from the discharge power supply is injected into the plasma through emission of primary electrons by the hot cathode and their acceleration in the plasma sheath around the filament [9]. Most of the applied discharge voltage drops across the sheath around the filament. As discussed later, these primary electrons are not carried all the way from the source to vessel wall or limiter of BETA, which acts as the anode. The electrons collected by the anode are the lower temperature plasma electrons. Nevertheless, the injected current has to have the same magnitude as the anode current. The current due to plasma ions is negligible [9] and is not taken into account for calculations here. The discharge volume in BETA device is $V_{\text {ol }} l_{\text {dis }} \sim 0.2 \mathrm{~m}^{3}$ and $\lambda_{m f p}$, the mean free path of ionizing primary electrons $\sim 100 \mathrm{~m}$. For $I_{p e}$ equal to discharge current of $5 A$ and using these values for $V o l_{d i s}$ and $\lambda_{m f p}$ we get the primary electron density $n_{p e} \sim 3.3 \times 10^{15}$ electrons $/ \mathrm{m}^{3}$. This value is at least two orders of magnitude greater than the electron density in the charge cylinder $\left(10^{12}-10^{13}\right.$ electrons/ $\mathrm{m}^{3}$ ) calculated from the measured electric field $E_{\perp}$.

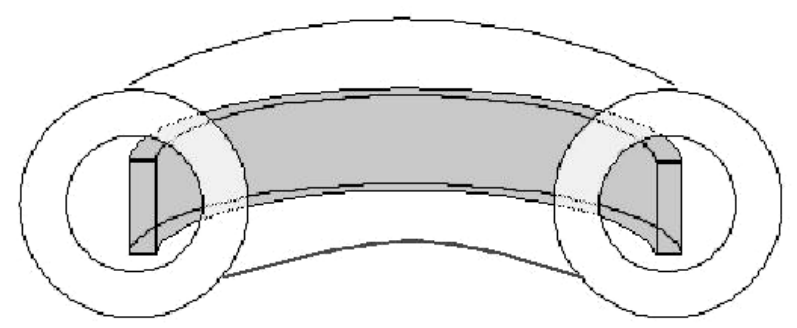

Figure 3. Schematic of the charge layer inside the plasma volume.

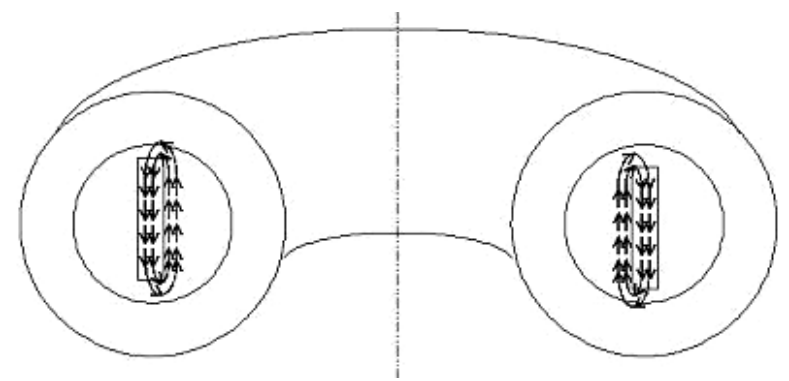

Figure 4. Schematic of the toroidal tube of convection cell. 


\subsubsection{Arc Current}

One can also estimate the number density of electrons participating in discharge current by another method. The primary electrons (injected by hot cathode) of energy $\sim 120 \mathrm{eV}$ are trapped by toroidal magnetic field although they drift due to curvature and grad B forces. It has been observed that the primary energetic electrons are not found beyond $2 \mathrm{~cm}$ in radial direction on the inboard and outboard sides of filament. They are also not found near the bottom side of the vessel due to downward drift. This implies that primary electrons do not reach the vessel wall of BETA. Hence, it is a good assumption to consider that the injected primary electrons are not directly participating in the discharge current. The functional role of primary electrons is to create ionization resulting in the formation of plasma and to excite neutral atoms of Ar. They spend the major portion of their energy through the processes of excitation of radiation levels in atoms.

In BETA, it is not possible to compensate the radial current from charge cylinder to the vessel wall or limiter due to classical diffusion [12-15]. On the other hand, electron flow parallel to the toroidal magnetic field form counter streams due to two sided injection of energetic electrons on the field lines that intercept filament and therefore do not contribute to the current. The single particle drifts (due to gradient and curvature of toroidal magnetic field and $\boldsymbol{E} \times \boldsymbol{B}$ drift) are insufficient in transporting the charge from filament region to the vessel wall. Therefore the cross-field charge transport is carried out by an anomalous mechanism initiated by the turbulence in the plasm [12-15].

Once plasma is created, the plasma electrons carry the discharge current across the toroidal magnetic field, enabled by anomalous perpendicular diffusion. The radial velocity of these electrons $v_{r}$ is $\sim k_{\theta} \varphi / B$, where $k_{\theta}$ is the poloidal wavevector of the dominant plasma fluctuations mode and $\varphi$ is amplitude of its electrostatic potential. For fluctuations in BETA, the range of poloidal wavevectors [11] is $-100 \mathrm{~m}^{-1} \leq k_{\theta} \leq 200 \mathrm{~m}^{-1}$. The value of poloidal wavevector $k_{\theta}$ for the dominant mode is $\sim 30 \mathrm{~m}^{-1}$. For the work reported in this paper, $B=0.02 \mathrm{~T}$ and $\varphi \sim 1 \mathrm{~V}$, resulting in radial velocity $v_{r}$ of $\sim 9.4 \times 10^{3} \mathrm{~m} / \mathrm{s}$. We note here that $v_{r}$ is much less that the velocity of the injected electrons at the plasmasheath boundary around the filament.

For $I_{d i s}=n_{p e} e v_{r} A_{w}=5 \mathrm{~A}$ and area of the vessel wall $A_{w}$ $=2.7 \mathrm{~m}^{2}$, the calculated value of $n_{p e} \sim 4.8 \times 10^{15}$ electrons $/ \mathrm{m}^{3}$. This value of primary electrons density $n_{p e}$ is of the order of value estimated in preceding section by the method of using the injected current is due to primary electrons. Further, $n_{p e}$ is just about $10 \%$ of the peak plasma density implying that only tail energy distribution of plasma electrons is sufficient to enable discharge current between the hot filament cathode of tungsten and the anode of vessel wall.

\subsection{Resolving Discrepancy between Measured and Injected Charge Density}

The discrepancy between the observed primary electron density (estimated in Section 4.1) and the calculated injected electron density (estimated in Sections 4.2.1 \& 4.2.2) in the charge cylinder implies that only a fraction of injected energetic electrons is retained as space charge of the charge cylinder. In fact, electron density in the charge cylinder is two orders of magnitude smaller than the density of injected electrons. The argument that most of the injected electrons, about $99 \%$, are lost due to the vertical drift is not supported by the observations. It has been observed experimentally that the negative potential well has a limited spatial extent in vertical direction and is receded from the vessel wall. The loss path of primary electrons to the vessel wall, along the vertically downward direction due to drifts, is not observed in the experiments. This implies that the charge cylinder, made up of primary electrons, does exist. Therefore, if there is a massive loss of injected electrons from the charge cylinder, it has to be by alternative processes, other than the drifts introduced by toroidal magnetic field. A reasonable explanation is that significant charge neutralization takes place as a consequence of inward ion diffusion towards the charge cylinder. This leads to significant reduction of electric field in the charge, i.e., $E_{\perp}$ to $500 \mathrm{~V} / \mathrm{m}$ as against the value $E_{\perp} \sim 10^{4} \mathrm{~V} / \mathrm{m}$ for the situation when electric field is sustained by the injected charge density (estimated in Sections 4.2.1 \& 4.2.2).

\subsection{Equilibrium of Charge Cylinder}

We now consider the dynamics of the observed charge cylinder that is independent of interaction of primary electrons with the surrounding plasma and all pervading neutral atoms. To a large extent, charge neutralization plays a considerable role in reducing the non-neutral component of plasma and consequently the magnitude of electric field inside the charge cylinder. The interaction with neutrals is through ionization and radiation. However, these interactions do not explain the existence of structure, which is isolated from the vessel wall and suspended in the plasma. To achieve isolation from the vessel wall, it is essential that the vertical drift of primary electrons is either cancelled or is converted into a poloidal drift at the extreme ends of the charge cylinder. Alternatively, a sink for primary electrons has to be placed at the bottom end of the cylinder. A sink is conceivable in terms of the filament support holders, which form a shadow region for the primary electrons. Against this hypothesis is the bias condition of these holders that are negatively biased to the same voltage as discharge 
voltage. So there cannot be any direct interception loss unless a mechanism of energization of primary electrons to energy greater than discharge voltage is invoked. The energization of only a minuscule number of primary electrons is possible by any mechanism. This is because only a small fraction of primary electrons can gain energy at the expense of energy residing in the remaining part of primary electrons through a multiple processes of exciting electrostatic potential waves in the charge cylinder. So such an explanation would not be suited to the bulk of primary electrons.

It seems then that explanation has to be in terms of that there are two drifts in the circulation pattern for primary ionizing electrons. In the purely toroidal magnetic field, these electrons exhibit a certain vertical drift due to gradient and curvature of the toroidal magnetic field. This drift forms the vertical leg of the circulation loop. The primary electrons also suffer $\boldsymbol{E} \times \boldsymbol{B}$-drift along the equipotential contours. This $\boldsymbol{E} \times \boldsymbol{B}$-drift enables the closing path of the circulation pattern. The combined effect of these two drifts is that $\boldsymbol{E} \times \boldsymbol{B}$ flow of the accumulated space charge will balance the charge accumulation due to the vertical drift of primary electrons as shown in Figure 4.

Following the preceding argument, we propose that there exists a stationary charge density distribution in the charge cylinder, satisfying the charge continuity equation. The stationary charge density distribution requires that the divergence term in continuity equation vanishes $\nabla \cdot J$ $=0$. To obtain a self-consistent solution, the continuity equation and Poisson's equation are solved simultaneously.

$$
\begin{gathered}
\frac{\partial \rho}{\partial t}+\nabla \cdot J=0 \\
\nabla^{2} \phi=-\frac{\rho}{\varepsilon_{0}}
\end{gathered}
$$

where

$$
J=\left[\rho \frac{\left(\hat{\mathrm{e}}_{\phi} \times \nabla \phi\right)}{B}+n_{p e} \mathrm{e} v_{D} \hat{\mathrm{e}}_{y}\right]
$$

$J$ is the current density, $\rho$ is the charge density, $n_{p e}$ is the number density of the primary ionizing electrons in the observed stationary charge cylinder, $v_{D}$ is the drift of monoenergetic electrons, $\phi$ is the space charge potential, $\varepsilon_{0}$ is the permittivity of free space and $\hat{e}_{\phi}$ and $\hat{e}_{y}$ are unit vectors in toroidal and poloidal directions. First term in the Equation (3) is the current due to $\boldsymbol{E} \times \boldsymbol{B}$ drift of the primary ionizing electrons and second term is their vertical current. The magnitude of the drift for the monoenergetic electrons leaving the filament is $v_{D}=2 V_{d i s} / B R$, where $V_{\text {dis }}$ is the discharge voltage, and $R=0.45 \mathrm{~m}$ is the radius of curvature of toroidal field. In order that $\nabla \cdot J=0$ for primary electrons

$$
\frac{\rho \phi}{a B}=\frac{2 n_{p e} e V_{D i s}}{B R}
$$

where $a$ is the minor radius of plasma torus

$$
\frac{\phi}{a^{2}}=\frac{\rho}{\varepsilon_{0}}
$$

Combining Equations (4) and (5), we estimate the required electric field for this stationary charge distribution constituting the charge cylinder viz.

$$
E \sim \sqrt{2 \frac{n_{p e} e V_{D i s}}{\varepsilon_{0}} \frac{a}{R}}
$$

For $n_{p e} \sim 10^{12}$ electrons $/ \mathrm{m}^{3}, V_{D i s}=120 \mathrm{~V}, a=0.9 \mathrm{~m}$ and $R=0.45 \mathrm{~m}$, the electric field required to set primary ionizing electrons circulating in the $\boldsymbol{E} \times \boldsymbol{B}$ direction is $\sim 300 \mathrm{~V} / \mathrm{m}$. As described earlier, the electric field estimated from the potential contour maps around the charge cylinder is $\sim 500 \mathrm{~V} / \mathrm{m}$. Thus, these calculations indicate that the self electric field set up by the charge cylinder enables a sufficient $\boldsymbol{E} \times \boldsymbol{B}$ drift for primary electrons to generate a charge cylinder in equilibrium. For $V_{D i s}=120$ $\mathrm{V}, B=0.02 \mathrm{~T}$ and $R=0.45 \mathrm{~m}$, the vertical drift $v_{D} \sim 2.67$ $\times 10^{4} \mathrm{~m} / \mathrm{s}$. On the other hand, for $E=500 \mathrm{~V} / \mathrm{m}$ and $B=$ $0.02 \mathrm{~T}$, the $\boldsymbol{E} \times \boldsymbol{B}$ drift $v_{E} \sim 2.5 \times 10^{4} \mathrm{~m} / \mathrm{s}$. This drift is directed along the equipotential surface. It may be noted that $v_{D}$ and $v_{E}$ are of the same order and their ratio is independent of the toroidal magnetic field.

Putting the vertical drift due to toroidal magnetic field and $\boldsymbol{E} \times \boldsymbol{B}$ drift of the primary electrons together, a broad picture that emerges is that the charge cylinder consists of a toroidal tube of convection cell, as shown in Figure 4, with cylinder as the vertical axis. Since the potential well depth is well below the discharge voltage (i.e., 120 $\mathrm{V})$, the electrons in the convection cell are those, which have lost their energy through ionization and radiation processes by electron-neutral collisions.

\section{Summary}

The plasma in BETA device is produced by a hot filament cathode discharge. The hot filament cathode injects charge into the plasma. Therefore the plasma embeds a charge cylinder of $\sim 3 \mathrm{~cm}$ thickness and $\sim 14 \mathrm{~cm}$ height and located $\sim 2 \mathrm{~cm}$ towards corresponding to the filament location in the poloidal cross-section. The energetic primary ionizing electrons injected by the hot filament cathode create this charge cylinder. The comparison of the injected and observed charge density indicates discrepancy between them. The observed value of charge density shows that only a small amount of residual space charge exists that is responsible for the creation of a charge cylinder. This electric field functions much in the 
similar fashion as in biased electrode experiments of tokamaks [16,17] and biased ring experiment in BETA $[18,19]$. The major difference between the excited electric field by the filament, aiding in the plasma equilibrium in BETA, and enhanced confinement mode experiments on tokamak and BETA is that, in the latter electric fields are excited from the boundary while in the present case it is excited from the core of plasma. Examining the equilibrium configuration of the charge cylinder indicates that it is made up of the self-consistent circulation pattern formed by the vertically drifting and poloidally flowing primary electrons. This self-consistency is in adjusting the poloidal drift to the vertical toroidal drift of the trapped primary electrons.

\section{REFERENCES}

[1] S. Roy, B. P. Pandey, J. Poggie and D. V. Gaitonde, "Modeling Low Pressure Collisional Plasma Sheath with Space-Charge Effect," Physics of Plasmas, Vol. 10, No. 6, 2003, pp. 2578-2585. doi:10.1063/1.1572491

[2] R. Iiyoshi, "Numerical Study on Emission Characteristics of a Point Cathode Electron Gun: Determination of Space Charge using Random Emitting Conditions," e-Journal of Surface Science and Nanotechnology, Vol. 4, 2006, pp. 339-344. doi:10.1380/ejssnt.2006.339

[3] R. Iiyoshi, "Numerical Analysis of Space Charge in a Point Cathode Thermionic Emission Gun," e-Journal of Surface Science and Nanotechnology, Vol. 1, 2003, pp. 147-151. doi:10.1380/ejssnt.2003.147

[4] M. S. Benilov, "Understanding and Modelling PlasmaElectrode Interaction in High-Pressure Arc Discharges: A Review," Journal of Physics D: Applied Physics, Vol. 41, No. 14, 2008, Article ID: 144001. doi:10.1088/0022-3727/41/14/144001

[5] M. S. Benilov and A. Marotta, "A Model of the Cathode Region of Atmospheric Pressure Arcs," Journal of Physics D: Applied Physics, Vol. 28, No. 9, 1995, pp. 18691882. doi: $10.1088 / 0022-3727 / 28 / 9 / 015$

[6] J. R. Ahn and C. J. Park, "Computer Simulation of Electron and Ion Trajectories in Electron-Impact Ion Sources of a Quadrupole Mass Spectrometer," Nuclear Instruments \& Methods in Physics Research Section A: Accelerators Spectrometers Detectors and Associated Equipment, Vol. 645, No. 1, 2011, pp. 345-349.

[7] M. R. B. Ghadikolaee, B. Fateh and E. T. Ghadikolaee, "Design and Study of an Enhanced Filament Ion Source," Vacuum, Vol. 86, No. 1, 2011, pp. 44-47. doi:10.1016/j.vacuum.2011.03.028

[8] H. Somacal, H. Huck, D. E. DiGregario, J. O. F. Niello and M. Igarzabal, "Simulations of Electron Trajectories under the Influence of an Array of Permanent Magnets in a Compact Ion Source," Nuclear Instruments \& Methods in Physics Research Section A: Accelerators Spectrometers Detectors and Associated Equipment, Vol. 490, No. 1, 2002, pp. 9-15.

[9] R. Kaur and S. K. Mattoo, "Inhomogeneous Electron Emission from a Hot Filament in a Toroidal Magnetic Field," Plasma Sources Science and Technology, Vol. 18, No. 1, 2009, Article ID: 015993.

[10] R. Kaur, A. S. Sree, A. K. Singh and S. K. Mattoo, "Life Cycle of Density Structures in a Simple Magnetized Torus," New Journal of Physics, Vol. 12, 2010, pp. 103013103018. doi:10.1088/1367-2630/12/10/103013

[11] R. Kaur, A. K. Singh, R. Singh, A. S. Sree and S. K. Mattoo, "Territorial Characteristics of Low Frequency Electrostatic Fluctuations in a Simple Magnetized Torus," Physics of Plasmas, Vol. 18, No. 1, 2011, pp. 1210912111. doi:10.1063/1.3551514

[12] R. Singh, S. Mahajan and K. Avinash, "Equilibrium and Fluctuations in a Plasma Confined in a Pure Toroidal Field," Physical Review Letters, Vol. 77, No. 8, 1996, pp. 1504-1507. doi:10.1103/PhysRevLett.77.1504

[13] S. Mahajan, R. Singh and K. Avinash, "Theory of Plasma Confinement in Devices with Pure Toroidal Field," Physics of Plasmas, Vol. 4, No. 7, 1997, pp. 2612-2618. doi: $10.1063 / 1.872349$

[14] K. Rypdal, E. Gronvoll, F. J. Oynes, A. Fredriksen, R. J. Armstrong, J. Trulsen and H. L. Pecseli, "Confinement and Turbulent Transport in a Plasma Torus with no Rotational Transform," Plasma Physics and Controlled Fusion, Vol. 36, No. 7, 1994, pp. 1099-1114. doi: $10.1088 / 0741-3335 / 36 / 7 / 002$

[15] R. Van Nieuwenhove and G. Van Oost, "Charge Neutralization by Poloidal Plasma Rotation," Plasma Physics and Controlled Fusion, Vol. 34, No. 5, 1992, pp. 873-880. doi:10.1088/0741-3335/34/5/015

[16] E. Wagner, et al., "Regime of Improved Confinement and High Beta in Neutral-Beam-Heated Divertor Discharges of the ASDEX Tokamak," Physical Review Letters, Vol. 49, No. 19, 1982, pp. 1408-1412. doi:10.1103/PhysRevLett.49.1408

[17] E. J. Strait, et al., "Enhanced Confinement and Stability in DIII-D Discharges with Reversed Magnetic Shear," Physical Review Letters, Vol. 75, No. 24, 1995, pp. 44214424. doi:10.1103/PhysRevLett.75.4421

[18] K. K. Jain, “Observation of Improved Behavior by Electrode Biasing of a Toroidal Plasma Having no Poloidal Magnetic Field," Physical Review Letters, Vol. 70, No. 6, 1993, pp. 806-809. doi:10.1103/PhysRevLett.70.806

[19] K. K. Jain, "Poloidal Plasma Rotation and Its Effect on Fluctuations in a Toroidal Plasma," Nuclear Fusion, Vol. 36, No. 12, 1996, pp. 1661-1669. doi:10.1088/0029-5515/36/12/I06 